\title{
COLLINEATIONS IN GENERALIZED SPACES
}

JACK LEVINE

1. Introduction. In this paper we derive simplified forms of the equations defining the infinitesimal projective and affine collineations of a generalized space of paths $H_{n}$. The complete solutions of these equations are then obtained for the two-dimensional spaces $H_{2}$. It is found that there are nine types of $H_{2}$ admitting continuous real groups $G_{r}$ of projective collineations (P. C.), and also nine types admitting affine collineations (A. C.). In each case the maximum number of parameters $r$ is three. In these classifications all solutions giving $H_{2}$ which reduce to ordinary affinely connected or projectively connected spaces are excluded. (These were obtained in [8].) ${ }^{1}$

A generalized space of paths $H_{n}$ of $n$ dimensions is characterized by a set of $n$ functions $H^{i}(x, d x)$ which are homogeneous of the second degree in $d x^{1}, \cdots, d x^{n}$. The paths are defined by

$$
d^{2} x^{i} / d s^{2}+H^{i}(x, d x / d s)=0 .
$$

Such spaces have been discussed by Knebelman [6] and Douglas $[4 ; 5]$ and in $[6]$ the theory of collineations in $H_{n}$ has been introduced. Further results on collineations in generalized spaces may be found in $[2 ; 7 ; 10 ; 11 ; 12 ; 13 ; 14 ; 15]$.

The components of affine connection $\Gamma_{j k}^{t}(x, d x)$ of an $H_{n}$ are defined by

$$
\Gamma_{j k}^{i}=\frac{1}{2} \frac{\partial^{\imath} H^{i}}{\partial\left(d x^{j}\right) \partial\left(d x^{k}\right)},
$$

and are homogeneous of degree zero in the $d x$. In terms of the $\Gamma$ 's the paths (1.1) have the familiar form

$$
d^{2} x^{i} / d s^{2}+\Gamma_{j k}^{i}\left(d x^{j} / d s\right)\left(d x^{k} / d s\right)=0 .
$$

In case the $H^{i}$ are second degree polynomials in the $d x$, the $H_{n}$ reduces to an ordinary affine space of paths $A_{n}$.

Two affine connections $\Gamma_{j k}^{t}(x, d x), \Gamma_{j k}^{\prime \prime}(x, d x)$ will possess the same paths if

$$
\Gamma_{j k}^{\prime i}=\Gamma_{j k}^{i}+\delta_{j \phi \cdot k}^{i}+\delta_{k}^{i} \phi \cdot j+\phi \cdot j k d x^{i}
$$

Presented to the Society, April 22, 1950 and April 29, 1950; received by the editors April 15, 1950.

${ }^{1}$ Numbers in brackets refer to the bibliography at the end of the paper. 
where $\phi(x, d x)$ is an arbitrary function homogeneous of the first degree in the $d x$ [6]. (Subscripts following a dot will indicate differentiations with respect to the $d x$.)

Elimination of the $\phi_{._{i}}$ in (1.4) leads to the projective relation $\Pi_{j k}^{\prime i}=\Pi_{j k}^{i}$, where the components $\Pi_{j k}^{i}$ of projective connection are defined by

$$
\Pi_{j k}^{i} \equiv \Gamma_{j k}^{i}-\frac{1}{n+1}\left(\delta_{j}^{i} \Gamma_{a k}^{a}+\delta_{k}^{i} \Gamma_{a j}^{a}+\Gamma_{a j k}^{a} d x^{i}\right) ; \quad \Pi_{i j}^{i}=0 .
$$

The components $\Gamma_{i j k}^{h} \equiv \Gamma_{i j \cdot k}^{h}$ are those of a tensor symmetric in $i, j, k$.

A collineation in an $H_{n}$ is defined as a point transformation $\bar{x}^{i}$ $=\bar{x}^{i}(x)$ which transforms paths into paths. In case the path-parameter $(s)$ is preserved we have an A. C., otherwise a P. C. A form of the path equations (1.3) unchanged by arbitrary parameter transformations is given by [6],

$$
\left(d^{2} x^{i}+\Gamma_{j k}^{i} d x^{j} d x^{k}\right) d x^{h}-\left(d^{2} x^{h}+\Gamma_{j k}^{h} d x^{j} d x^{k}\right) d x^{i}=0 .
$$

The conditions on an $H_{n}$ for it to admit a continuous $G_{r}$ of P. C. were obtained by Knebelman [6], through the use of the infinitesimal P. C. determined by

$$
\bar{x}^{i}=x^{i}+\xi^{i}(x) \delta t
$$

These conditions are

$(1.8) \xi_{; j k}^{i}+\xi^{h} B_{j k h}^{i}+\xi_{; m}^{h} \Pi_{j k h}^{i} d x^{m}-\left(\delta_{j}^{i} \psi_{; k}+\delta_{k}^{i} \psi_{; j}\right)=0,\left(\Pi_{i j k}^{h} \equiv \Pi_{i j \cdot k}^{h}\right)$, where a semicolon indicates the (projective) covariant derivative, for example,

$$
T_{j ; k}^{i}(x, d x)=\partial T_{j}^{i} / \partial x^{k}+T_{j}^{h} \Pi_{h k}^{i}-T_{h}^{i} \Pi_{j k}^{h}-T_{j \cdot h}^{i} \Pi_{m k}^{h} d x^{m}
$$

Also,

$$
\begin{aligned}
B_{j k h}^{i} \equiv & \frac{\partial \Pi_{j k}^{i}}{\partial x^{h}}-\frac{\partial \Pi_{j h}^{i}}{\partial x^{k}}+\Pi_{j k}^{m} \Pi_{m h}^{i}-\Pi_{j h}^{m} \Pi_{m k}^{i} \\
& +\left(\Pi_{a k}^{m} \Pi_{j h m}^{i}-\Pi_{a h}^{m} \Pi_{j k m}^{i}\right) d x^{a} \\
\psi_{; i}= & (1 /(n+1)) \xi_{; a i}^{a} .
\end{aligned}
$$

If an $H_{n}$ admits the $r$ linearly independent infinitesimal P. C. with generators $X_{\alpha} f=\xi_{\alpha \mid}^{i} \partial f / \partial x^{i}(\alpha=1, \cdots, r)$, then it is known to admit the finite continuous (local) group $G_{r}=\left[X_{1}, X_{2}, \cdots, X_{r}\right]$ generated 
by the $X_{\alpha} f[2 ; 6 ; 12]$.

It has been shown $[2 ; 12]$ that $(1.8)$ can be expressed in the form

$$
\Delta \Pi_{j k}^{i}=0,
$$

where $\Delta$ is the Lie derivative operator

$$
\Delta \Pi_{j k}^{i}=\lim _{\delta t \rightarrow 0} \frac{\Pi_{j k}^{i}(\bar{x}, d \bar{x})-\bar{\Pi}_{j k}^{i}(\bar{x}, d \bar{x})}{\delta t}
$$

with respect to the infinitesimal transformation (1.7). For a general discussion of this operator, references $[3 ; 11 ; 12 ; 14 ; 15]$ may be consulted.

When written in expanded form (1.8) or (1.12) is

$$
\begin{gathered}
\frac{\partial^{2} \xi^{i}}{\partial x^{j} \partial x^{k}}+\Pi_{j m}^{i} \frac{\partial \xi^{m}}{\partial x^{k}}+\Pi_{m k}^{i} \frac{\partial \xi^{m}}{\partial x^{j}}-\Pi_{j k}^{m} \frac{\partial \xi^{i}}{\partial x^{m}}+\Pi_{j k m}^{i} \frac{\partial \xi^{m}}{\partial x^{h}} d x^{h} \\
+\xi^{m} \frac{\partial \Pi_{j k}^{i}}{\partial x^{m}}=\frac{1}{n+1}\left(\delta_{j}^{i} \frac{\partial^{2} \xi^{h}}{\partial x^{h} \partial x^{k}}+\delta_{k}^{i} \frac{\partial^{2} \xi^{h}}{\partial x^{h} \partial x^{j}}\right) .
\end{gathered}
$$

If in (1.14) the $\Pi_{j \mathbf{k}}^{i}$ be replaced by $\Gamma_{j \mathbf{k}}^{i}$ and the right member by 0 , we obtain the equations of $\mathrm{A}$. C.

$$
\begin{aligned}
\frac{\partial^{2} \xi^{i}}{\partial x^{j} \partial x^{k}} & +\Gamma_{j m}^{i} \frac{\partial \xi^{m}}{\partial x^{k}}+\Gamma_{m k}^{i} \frac{\partial \xi^{m}}{\partial x^{j}}-\Gamma_{j k}^{m} \frac{\partial \xi^{i}}{\partial x^{m}} \\
& +\xi^{m} \frac{\partial \Gamma_{j k}^{i}}{\partial x^{m}}+\Gamma_{j k m}^{i} \frac{\partial \xi^{m}}{\partial x^{h}} d x^{h}=0 .
\end{aligned}
$$

These latter equations may also be written in terms of the Lie derivative as [3]

$$
\Delta \Gamma_{j k}^{i}=0 .
$$

2. Simplified form of collineation equations. To obtain a simplified form of these equations we consider first (1.12) and define quantities $P^{i}(x, d x)$ by

$$
P^{i} \equiv H^{i}-\frac{1}{n+1} F d x^{i}
$$

with

$$
F(x, d x)=H_{\cdot i}^{i}
$$

being homogeneous of first degree and $P^{i}$ of second degree in the $d x$. 
It is then easily shown that

$$
2 \mathrm{II}_{j k}^{i}=P_{\cdot j k}^{i}
$$

and

$$
2 \Delta \Pi_{j k}^{i}=\Delta\left(P_{\cdot j k}^{i}\right)=\left(\Delta P^{i}\right) \cdot j k,
$$

since the operators $\Delta$ and $\partial(d x)$ are here commutative $[2 ; 3 ; 10 ; 11]$.

It follows from the homogeneity in $d x$ of $\Delta \Pi_{j k}^{i}$ that

$$
\Delta P^{i}=\left(\Delta \Pi_{j k}^{i}\right) d x^{j} d x^{k},
$$

and thus the equations of $P$. C. can be expressed in the equivalent form

$$
\Delta P^{i}=0
$$

Equations (2.5) show also that $\Delta P^{i}$ are components of a contravariant vector.

In a similar manner it can be shown that the $A$. $C$. equations can be expressed as

$$
\Delta H^{i}=0,
$$

with $\Delta H^{i}$ also contravariant vector components.

To obtain the explicit form of $\Delta P^{i}$ we may use (1.4) and (2.5) or else the definition $\Delta P^{i}=\lim _{\delta t \rightarrow 0}\left[P^{i}(\bar{x}, d \bar{x})-\bar{P}^{i}(\bar{x}, d \bar{x})\right] / \delta t$. In the latter case we make use of the transformation equation $[4,12]$

$$
\begin{aligned}
\bar{H}^{i} & =H^{j} \frac{\partial \bar{x}^{i}}{\partial x^{j}}-\frac{\partial^{2} \bar{x}^{i}}{\partial x^{j} \partial x^{k}} d x^{j} d x^{k}, \\
\bar{F} & =F-2 \frac{\partial^{2} \bar{x}^{i}}{\partial x^{j} \partial x^{k}} \frac{\partial x^{k}}{\partial \bar{x}^{i}} d x^{j} .
\end{aligned}
$$

There is obtained

$$
\begin{aligned}
\Delta P^{i}= & \frac{\partial^{2} \xi^{i}}{\partial x^{j} \partial x^{k}} u^{j} u^{k}+\xi^{m} \frac{\partial P^{i}}{\partial x^{m}}+\frac{\partial \xi^{m}}{\partial x^{h}} \frac{\partial P^{i}}{\partial u^{m}} u^{h}-\frac{\partial \xi^{i}}{\partial x^{m}} P^{m} \\
& -\frac{2}{n+1} \frac{\partial^{2} \xi^{h}}{\partial x^{h} \partial x^{a}} u^{a} u^{i}
\end{aligned}
$$

where $u^{i}=d x^{i}$.

It is to be noted that the $P^{i}$ satisfy the identity

$$
P_{\cdot i}^{i}=0 \text {. }
$$


Also, corresponding to the projective change (see (1.4))

$$
H^{\prime i}=H^{i}+\phi d x^{i}, \quad F^{\prime}=F+(n+1) \phi,
$$

we have

$$
P^{\prime i}=P^{i} \text {. }
$$

To obtain the explicit form of $\Delta H^{i}$ we proceed as above, making use of (2.8). We find

$$
\Delta H^{i}=\frac{\partial^{2} \xi^{i}}{\partial x^{j} \partial x^{k}} u^{i} u^{k}+\frac{\partial H^{i}}{\partial x^{m}} \xi^{m}+\frac{\partial H^{i}}{\partial u^{m}} \frac{\partial \xi^{m}}{\partial x^{h}} u^{h}-\frac{\partial \xi^{i}}{\partial x^{m}} H^{m}
$$

This expression has also been obtained by Kosambi [7] in a different way.

It follows from (2.1) that in order for a P. C. to reduce to an A. C. the $\xi^{i}$ must satisfy besides (2.6) the further condition

$$
\Delta F=\xi^{i} \frac{\partial F}{\partial x^{i}}+\frac{\partial F}{\partial u^{i}} \frac{\partial \xi^{i}}{\partial x^{j}} u^{j}+2 \frac{\partial^{2} \xi^{i}}{\partial x^{i} \partial x^{j}} u^{j}=0 .
$$

The A. C. can thus be obtained either by solving (2.7) directly or else by solving the system (2.6) and (2.15). Since we wish to find the P. C. also we have used the latter method.

3. Collineations in $H_{2}$. The results of the preceding section are now applied to the two-dimensional spaces $H_{2}$. Equations (2.6), considered in the unknowns $P^{i}$, are solved to obtain all spaces $H_{2}$ which admit real groups $G_{r}$ of P. C. As in [8] the $\xi^{i}$ are obtained from the classification of Lie [9] giving all real continuous groups $G_{r}$ in two variables. We also put $x^{1}, x^{2}=x, y$ and $u^{1}, u^{2}=u, v$.

Now if $n=2,(2.11)$ shows that $d P=-P^{2} d u+P^{1} d v$ is an exact differential with

$$
P^{1}=\partial P / \partial v, \quad P^{2}=-\partial P / \partial u .
$$

Also, $P$ is homogeneous of the third degree in $u, v$. The $\mathrm{II}_{j k}^{i}$ can then be expressed in terms of $P$ which remains as the basic function to be determined in each case. If it is desired to obtain the $H^{i}$, we use (2.1) in which $F$ is now considered as an arbitrary function homogeneous of the first degree in the $d x^{i}$.

To find the A. C. determined by a particular $G_{r}$ we solve (2.15) for $F$, and using the solutions for $P^{1}, P^{2}$ as given by (3.1) through the P. C. solution we obtain the $H^{i}$ from (2.1).

To illustrate the general procedure we find the spaces admitting the $G_{3}[p, q,(y+c x) p+(c y-x) q](c$ an arbitrary constant) as a group of 
collineations. Here, as later, $p=\partial f / \partial x, q=\partial f / \partial y$.

We obtain first the P. C. The generators $X_{1} f=p, X_{2} f=q$ show by (2.6) that $P^{i}$ is a function of $u$ and $v$ only. On substituting the third generator components $\xi_{3 \mid}^{1}=y+c x, \xi_{3 \mid}^{2}=c y-x$ in (2.6) we have

$$
\begin{aligned}
& v \frac{\partial P^{1}}{\partial u}-u \frac{\partial P^{1}}{\partial v}=-c P^{1}+P^{2}, \\
& v \frac{\partial P^{2}}{\partial u}-u \frac{\partial P^{2}}{\partial v}=-P^{1}-c P^{2},
\end{aligned}
$$

with the solutions

$$
\begin{aligned}
& P^{1}=u^{2}(f w+g)\left(1+w^{2}\right)^{1 / 2} e^{c \tan ^{-1} w}, \\
& P^{2}=u^{2}(g w-f)\left(1+w^{2}\right)^{1 / 2} e^{c \tan ^{-1} w}, \quad w=v / u,
\end{aligned}
$$

where $f, g$ are arbitrary constants not both zero.

From (3.1) we then obtain $3 g-c f=0$, and the value of $P$,

$$
\begin{array}{r}
P=\frac{f}{3} u^{3}\left(1+w^{2}\right)^{1 / 2} e^{c \tan ^{1} w}=\frac{f}{3}\left(d x^{2}+d y^{2}\right)^{1 / 2} e^{c \tan ^{-1} d y / d x}, \\
f \neq 0 .
\end{array}
$$

The components $\Pi_{j k}^{i}$ of projective connection of the corresponding $\mathrm{H}_{2}$ can now be found by (2.3) and (3.3) with $g=c f / 3$.

To determine the spaces admitting the above $G_{3}$ as A. C. we must solve (2.15) for $F$. From $X_{1}$ and $X_{2}$ we have $F(u, v)$, and $X_{3}$ gives the single condition

$$
v \frac{\partial F}{\partial u}-u \frac{\partial F}{\partial v}+c F=0
$$

Hence $F=d u\left(1+w^{2}\right)^{1 / 2} e^{c \tan ^{-1} w}$ with $d$ an arbitrary constant.

The values of $H^{1}$ and $H^{2}$ of the $H_{2}$ admitting the $G_{3}$ as A. C. are thus

$$
\begin{aligned}
& H^{1}=(a d y+b d x)\left(d x^{2}+d y^{2}\right)^{1 / 2} e^{c \tan ^{-1} d y / d x}, \\
& H^{2}=(b d y-a d x)\left(d x^{2}+d y^{2}\right)^{1 / 2} e^{c \tan ^{-1} d y / d x} .
\end{aligned}
$$

The affine connection components can then be found from (1.2). In the following two lists which represent all possible $H_{2}$ admitting real groups of P. C. and A. C. respectively the reducible $\mathrm{H}_{2}$ have been excluded. In the case of $\mathrm{P}$. C. such spaces result from functions $P$ which are cubic polynomials in $d x, d y$, so that the $\Pi_{j k}^{i}$ are functions of position ( $x$ and $y$ ) only. 
The reducible $H_{2}$ admitting A. C. are such that the $H^{i}$ are seconddegree polynomials in $d x, d y$.

Each of the 9 groups in the two lists is a complete group of collineations, that is, it is the group of maximum number of parameters which the associated $\mathrm{H}_{2}$ will admit. For each of the six $G_{3}$ spaces this follows, since otherwise the $H_{2}$ would be reducible. That the $G_{1}$ and $G_{2}$ groups are complete follows from similar results of [8].

$\mathrm{H}_{2}$ spaces admitting complete groups of projective collineations

$$
[p] \text {, }
$$

$$
P=P(y ; d x, d y) \text {. }
$$

$$
[p, q] \text {, }
$$

$$
P=P(d x, d y) \text {. }
$$

$$
[p, x p+y q]
$$

$$
P=(1 / y) Q(d x, d y) \text {. }
$$

$$
[p, q, x p+c y q]
$$

$$
P=k[(c-1) /(1-2 c)](d x)^{3}(d y / d x)^{(2-c) /(1-c)} \text {. }
$$

$[p, q, x p+(x+y) q]$

$$
P=k(d x)^{3} e^{-d y / d x} \text {. }
$$

$$
\left[p, x p+y q, x^{2} p+\left(2 x y+y^{2}\right) q\right] \text {, }
$$

$$
P=(d x)^{3}\left(1+\frac{d y}{d x}\right)\left[k\left(1+\frac{d y}{d x}\right)^{1 / 2}+\frac{2}{3}\left(2+\frac{d y}{d x}\right)\right] \text {. }
$$

$$
[p, q,(y+c x) p+(c y-x) q] \text {, }
$$

$$
P=k\left(d x^{2}+d y^{2}\right)^{1 / 2} e^{c \tan ^{-1} d y / d x} \text {. }
$$

$$
\begin{aligned}
& {\left[y p-x q,\left(1+\epsilon x^{2}\right) p+\epsilon x y q, \epsilon x y p+\left(1+\epsilon y^{2}\right) q\right](\epsilon= \pm 1),} \\
& P=k\left[\frac{\epsilon\left(d x^{2}+d y^{2}\right)+(x d y-y d x)^{2}}{x^{2}+y^{2}+\epsilon}\right]^{3 / 2}
\end{aligned}
$$

In [G1.1] and [G2.1] $P$ is an arbitrary function of its arguments as is $Q$ in [G2.2] (homogeneous of third degree in $d x, d y$ ), and in all other cases $k$ is an arbitrary nonzero constant.

$\mathrm{H}_{2}$ spaces admitting complete groups of affine collineations

[G1.1] $\quad H^{i}=H^{i}(y ; d x, d y)$.

[G2.1] $\quad H^{i}=H^{i}(d x, d y)$.

[G2.2] $\quad H^{i}=(2 / y) A^{i}(d x, d y)$. 
[G3.1] $\quad H^{1}=a(d x)^{2}(d y / d x)^{1 /(1-c)}, H^{2}=b d x d y(d y / d x)^{1 /(1-c)}$

[G3.2] $\quad H^{1}=2 a(d x)^{2} e^{-d y / d x}, \quad H^{2}=2(d x)^{2}[a(d y / d x)+b] e^{-d y / d x}$. $H^{1}=(2 / y)(d x)^{2}(1+2 a R)$,

[G3.3]

$$
H^{2}=-(2 / y)(d x)^{2}\left(1+2 a R+2 b R^{3}+2 R^{4}\right), R=\left(1+\frac{d y}{d x}\right)^{1 / 2} \text {. }
$$

$H^{1}=(a d y+b d x)\left(d x^{2}+d y^{2}\right)^{1 / 2} e^{c \tan ^{-1} d y / d x}$, $H^{2}=(b d y-a d x)\left(d x^{2}+d y^{2}\right)^{1 / 2} e^{c \tan ^{-1} d y / d x}$.

$$
H^{1}=(d x)^{2}\left[-2 \frac{x+y(d y / d x)}{x^{2}+y^{2}+\epsilon}\right.
$$

$$
\left.+\frac{1}{\left(x^{2}+\epsilon\right)^{1 / 2}} \frac{1}{\left(x^{2}+y^{2}+\epsilon\right)^{1 / 2}}(a T+b)\left(1+T^{2}\right)^{1 / 2}\right]
$$

$\left[\begin{array}{l}\mathrm{G} 3.5 \\ \mathrm{G} 3.6\end{array}\right]$

$$
\begin{aligned}
H^{2} & =(d x)^{2}\left[-2 \frac{x+y(d y / d x)}{x^{2}+y^{2}+\epsilon} \frac{d y}{d x}\right. \\
& \left.+\frac{(a T+b)\left(1+T^{2}\right)^{1 / 2}}{\left(x^{2}+\epsilon\right)^{3 / 2}}\left(\frac{b T-a}{a T+b}+\frac{x y}{\left(x^{2}+y^{2}+\epsilon\right)^{1 / 2}}\right)\right], \\
T & =\left[\left(x^{2}+\epsilon\right)(d y / d x)-x y\right] /\left(x^{2}+y^{2}+\epsilon\right)^{1 / 2} .
\end{aligned}
$$

Here $a, b, c$ are arbitrary constants, with $a, b$ not both zero. Also, in [G3.1] $b \neq 0$ if $c=1 / 2$, and $a \neq 0$ if $c=2$. The $A^{i}$ in [G2.2] are arbitrary functions homogeneous of second degree in $d x, d y$. Similar remarks apply to the $H^{i}$ of [G1.1] and [G2.1].

We summarize these results in the following theorem:

THEOREM 3.1. If a (nonreducible) two-dimensional generalized space $\mathrm{H}_{2}$ admits a real continuous group $G_{r}$ of projective or affine collineations then $r \leqq 3$. There are nine such complete groups of collineations, one $G_{1}$, two $G_{2}$, and six $G_{3}$.

\section{BIBLIOGRAPHY}

1. Ludwig Berwald, Untersuchung der Krummung allgemeiner metrischer Raume auf Grund des in ihnen herrschenden Parallelismus, Math. Zeit. vol. 25 (1928) pp. 40-73.

2. R. S. Clark, Projective collineations in a space of $K$-spreads, Proc. Cambridge Philos. Soc. vol. 41 (1945) pp. 210-223.

3. E. T. Davies, On the isomorphic transformations of a space of $K$-spreads, J. London Math. Soc. vol. 18 (1943) pp. 100-107.

4. Jesse Douglas, The general geometry of paths, Ann. of Math. vol. 29 (1928) pp. 154-166.

5. - System of $K$-dimensional manifolds in an $N$-dimensional space, Math. Ann. vol. 105 (1931) pp. 527-564. 
6. M. S. Knebelman, Collineations and motions in generalized spaces, Amer. J. Math. vol. 51 (1928) pp. 527-564.

7. D. D. Kosambi, Collineations in path-space, J. Indian Math. Soc. N.S. vol. 1 (1934) pp. 69-72.

8. Jack Levine, Classification of collineations in projectively and affinely connected spaces of two dimensions, Ann. of Math. vol. 52 (1950) pp. 465-477.

9. Sophus Lie and Friedrich Engel, Theorie der Transformationsgruppen, vol. 3, Leipzig, Teubner, 1893, pp. 71-73, 379-380.

10. Buchin $\mathrm{Su}$, On the isomorphic transformations of $K$-spreads in a Douglas space. I, Science Record vol. 2 (1947) pp. 11-19.

11. - On the isomorphic transformations of $K$-spreads in a Douglas space. II, Science Record vol. 2 (1948) pp. 139-146.

12. - Descriptive collineations in spaces of $K$-spreads, Trans. Amer. Math. Soc. vol. 61 (1947) pp. 495-507.

13. - A characteristic property of affine collineations in a space of $K$-spreads, Bull. Amer. Math. Soc. vol. 54 (1948) pp. 136-138.

14. Kentaro Yano, Lie derivatives in general space of paths, Proc. Imp. Acad. Tokyo vol. 21 (1945) pp. 363-371.

15. - Groups of transformations in generalized spaces, Tokyo, Akademeia Press, 1949.

North Carolina State College

\section{ON THE CONTINUITY OF PARAMETRIC LINEAR OPERATIONS ${ }^{1}$}

\section{B. J. PETTIS}

The proofs of the theorems asserting strong continuity for semigroups of linear operations in Banach spaces usually involve measurability and integrability of Banach-space-valued functions [2, pp. 183-184]. ${ }^{2} \mathrm{~A}$ theorem of this type in which the assumptions and the proof are purely topological is given below.

Let $G$ be both a topological space and an additive group, and let $H$ be a subset of $G$. For each $h$ in $H$ let $D(h)$ be all points $g$ in $H$ satisfying these two conditions: (1) $h-g \in H$, (2) for each open set $N_{g}$ about $g$ there is an open set $N_{h}$ about $h$ such that $h-g+\left(H \cap N_{\theta}\right)$ $\supset H \cap N_{h}$. Letting $X$ be a complex linear normed space, a set $\Gamma=[\gamma]$ of bounded complex linear functionals on $X$ is a total set for $E, E$ a subset of $X$, if $\|x\|=\sup [|\gamma(x)|, \gamma \in \Gamma]$ holds for every $x$ in the smallest linear subspace containing $E$. A function $T_{h}$ on $H$ to the

Presented to the Society, April 21, 1951; received by the editors May 29, 1950.

${ }^{1}$ This paper was written under Contract N7-onr-434, Task Order III, Navy Department (The Office of Naval Research).

${ }^{2}$ Numbers in brackets refer to the references at the end of the paper. 